TRANSACTIONS OF THE AMERICAN MATHEMATICAL SOCIETY

Volume 348, Number 10, October 1996

\title{
WIENER'S TEST FOR SPACE-TIME RANDOM WALKS AND ITS APPLICATIONS
}

\author{
YASUNARI FUKAI AND KÔHEI UCHIYAMA
}

\begin{abstract}
This paper establishes a criterion for whether a $d$-dimensional random walk on the integer lattice $\mathbf{Z}^{d}$ visits a space-time subset infinitely often or not. It is a precise analogue of Wiener's test for regularity of a boundary point with respect to the classical Dirichlet problem. The test obtained is applied to strengthen the harder half of Kolmogorov's test for the random walk.
\end{abstract}

\section{INTRODUCTION}

The purpose of this paper is to prove Wiener's test for space-time random walks on the $(d+1)$-dimensional Euclidean lattice $\mathbf{Z}^{d+1}$ and to illustrate its applicability with certain examples, especially by deducing Kolmogorov's test for random walks from Wiener's test. In the special case of simple random walk it provides a criterion for regularity of a minimal Martin boundary point (at infinity) with respect to the difference equation of heat conduction in unbounded domains of $\mathbf{Z}^{d+1}$.

The classical Wiener test is a geometric characterization of a regular boundary point to the Dirichlet problem for the Laplace operator in a Euclidean domain $D$. The criterion is given in terms of capacity that measures how thick the set $A=D^{c}$ (the complement of $D$ ) is at the boundary point. As is well understood, the classical potential theory can be duly translated in terms of Markov processes, and Wiener's test accordingly has a probabilistic counterpart. A natural analogue for a $d$-dimensional simple random walk $(d \geq 3)$ in particular is obtained by ItoMcKean [IM]: it tests an infinite set, $A$ say, for recurrence or transience, namely for the dichotomy of whether the random walk visits $A$ infinitely or only finitely many times. Lamperti $[\mathrm{Lm}]$ generalizes the test to a class of transient Markov processes by using the level sets of the Green function in place of the Euclidean balls by means of which the set $A$ is partitioned into suitable subsets to be measured with capacity. What we obtain in this paper is a precise analogue for the space-time walks in this sense.

The condition imposed on the Markov processes in [Lm] is a set of certain inequalities for the Green function, and is utterly not satisfied by the space-time walk. This condition immediately implies an inequality for potentials (hitting probabilities) that is implicitly used in [Lm]. In our proof for the space-time walk it is a main task to obtain a weak version of that inequality (Proposition 2); that its proof

Received by the editors May 10, 1995.

1991 Mathematics Subject Classification. Primary 60J15, 60J45, 31C20.

Key words and phrases. Wiener's test, random walk, Kolmogorov's test, discrete heat equation, regularity of a minimal Martin boundary point. 
is rather involved reflects the gap between the parabolic and the elliptic problems (see [GL] for the gap recognized in the analytic approach).

Evans and Gariepy [EG] established Wiener's test for the space-time Brownian motion (i.e., for the heat operator) and Uchiyama [U] by a quite different method: it is quite probabilistic in [U], while purely analytic in [EG]. The present approach is similar to that of [U]. We apply the local limit theorem to approximate the Green functions (transition probabilities of the random walk) by the Gaussian kernel, which makes it possible to follow the main lines of the proof given by [U]. We however need some auxiliary estimates or different arguments in several places where the local limit theorem alone is not enough. Some of them (especially those for Lemmas 2 and 5) are useful also in the Brownian case, making the corresponding part of the proof in $[\mathrm{U}]$ simpler and more natural.

The application of the test is a matter quite different from its proof. The problem now is that of evaluation of capacity, for which a variational formula is a principal tool. To make it easier we improve the test by introducing a finer partitioning, as in [U]. Its effectiveness is well revealed in the deduction of Kolmogorov's test from Wiener's test. In $[\mathrm{U}]$ we make use of the explicit form of the hitting distribution (called Fulks' measure) of the space-time Brownian motion to the heat sphere for such an application. In the present paper we can completely dispense with it by thoroughly exploiting the refined version.

The contents of the rest of this paper are: $\S 2$ Main results; $\S 3$ A local limit theorem; $\S 4$ An inequality for hitting probabilities; $\S 5$ Proof of Theorems 1 and 2; $\S 6$ Refinement; $\S 7$ Applications; $\S 8$ Evaluation of capacity; $\S 9$ Appendix.

\section{MAIn RESUlts}

Let $X_{k}$ be $\mathbf{Z}^{d}$-valued i.i.d. random variables defined on a probability space $(\Omega, \mathcal{F}, P)$. Put $S_{n}:=\sum_{k=1}^{n} X_{k}, n=1,2, \ldots$, and $S_{0}:=\mathbf{0}$. Throughout the paper, we suppose, unless stated otherwise, that $E\left\{X_{1}\right\}=0$ and for some $p>1$

$$
E\left\{\left|X_{1}\right|^{2}\left(\log ^{+}\left|X_{1}\right|\right)^{p}\right\}<\infty,
$$

where $\log ^{+} a=\log (\max \{a, 1\})$ and $E\{\cdot\}$ indicates the integration by $P$ and $|\cdot|$ the Euclidean length, and further that the random walk $\left(S_{n}\right)_{n=0,1,2, \ldots}$ is irreducible, i.e., for every $x \in \mathbf{Z}^{d}$ there is an $n$ such that $P\left\{S_{n}=x\right\}>0$. (We shall use the moment condition (2.1) to obtain an error-term estimate of the local limit theorem that will be applied throughout the paper (except in Section 4). It however does not seem crucial for Wiener's test at all. See Remark 1 after Theorem 2 below for more comments.) Put

$$
P_{n}(x):=P\left\{S_{n}=x\right\}, \quad x \in \mathbf{Z}^{d} .
$$

Let $Q$ stand for the covariance matrix of $X_{1}: Q_{i, j}=E\left\{X_{1}^{(i)} X_{1}^{(j)}\right\} .\left(X_{1}^{(i)}\right.$ is the $i$-th component of $X_{1}$.) Then define $B_{k}^{*}$, 'the $k$-th ball centered at the origin $\hat{0}:=(0, \mathbf{0})$,' by

$$
B_{k}^{*}:=\left\{(n, x) \in \mathbf{N} \times \mathbf{Z}^{d}: P_{n}(x) \geq\left(2 \pi 2^{k}\right)^{-d / 2}|Q|^{-1 / 2}\right\} .
$$

Here $\mathbf{N}=\{1,2,3, \ldots\}$ and $|Q|$ is the determinant of $Q$. Let $A$ be a subset of $\mathbf{Z}^{1+d}$. We denote by $h(\xi, A)$, the hitting probability of $A$ by the space-time random walk starting at $\xi=(n, x) \in \mathbf{Z}^{1+d}$ :

$$
h((n, x), A):=P\left\{\left(n+m, x+S_{m}\right) \in A \text { for some } m \geq 0\right\} .
$$


Let us write ' $A$ is hit i.o.' if the space-time walk

$$
\hat{S}_{n}:=\left(n, S_{n}\right)
$$

hits points of $A$ infinitely often, i.e., $\hat{S}_{n} \in A$ for infinitely many $n$.

Theorem 1. Let $A$ be a subset of $\mathbf{N} \times \mathbf{Z}^{d}$. Put $A_{k}^{*}:=A \cap\left[B_{k}^{*} \backslash B_{k-1}^{*}\right]$. Then

$$
P\{A \text { is hit i.o. }\}=1 \quad \text { if and only if } \sum_{k=0}^{\infty} h\left(\hat{0}, A_{k}^{*}\right)=\infty \text {. }
$$

Let $G(\xi, \eta)$ be the Green function of the space-time walk:

$$
G(\xi, \eta)=\sum_{n=0}^{\infty} P\left\{\xi+\hat{S}_{n}=\eta\right\}, \quad \xi, \eta \in \mathbf{Z}^{d+1} .
$$

If $A$ is a finite set that is not empty, we have the identity

$$
h(\xi, A)=\sum_{\eta \in A} G(\xi, \eta) e_{A}(\eta),
$$

where $e_{A}(\eta)$ is the probability that the space-time walk starting at $\eta$ escapes $A$ for all positive times: $e_{A}(\eta)=P\left\{\eta+\hat{S}_{n} \notin A\right.$ for all $\left.n \in \mathbf{N}\right\}$. Since for $\xi=(n, x)$

$$
\begin{aligned}
& G(\hat{0}, \xi)=P_{n}(x) \quad \text { if } \quad n \geq 0, \\
& =0 \quad \text { if } n<0 \text {, }
\end{aligned}
$$

our ball $B_{k}^{*}$ is actually defined as a sub-level set of $G(\hat{0}, \cdot)$. Let cap $(A)$ denote the capacity of $A$ :

$$
\operatorname{cap}(A):=\sum_{\eta \in A} e_{A}(\eta) \quad \text { if } \quad A \neq \emptyset
$$

and $\operatorname{cap}(\emptyset):=0$. Then the relation $(2.2)$ verifies that for any $A \subset B_{k}^{*} \backslash B_{k-1}^{*}$

$$
2^{-k d / 2} \operatorname{cap}(A) \leq \sqrt{(2 \pi)^{d}|Q|} \cdot h(\hat{0}, A) \leq 2^{-(k-1) d / 2} \operatorname{cap}(A) .
$$

Therefore the equivalence in Theorem 1 can be paraphrased as follows:

Corollary 1. Let $A$ and $A_{k}^{*}$ be as in Theorem 1. Then

$$
P\{A \text { is hit i.o. }\}=1 \text { if and only if } \sum_{k=1}^{\infty} 2^{-k d / 2} \operatorname{cap}\left(A_{k}^{*}\right)=\infty .
$$

This is a precise analogue of the classical Wiener test. For both the proof and applications it is convenient to introduce another ' $k$-th ball' as a substitute for $B_{k}^{*}$. Put

$$
\|x\|=\sqrt{x \cdot Q^{-1} x}
$$

where $Q^{-1}$ is the inverse matrix of $Q$. The new one is defined by means of the heat kernel

$$
p(t, u):=\frac{1}{(2 \pi t)^{d / 2}|Q|^{1 / 2}} \exp \left(-\frac{\|u\|^{2}}{2 t}\right) \quad\left(t \in(0,+\infty), u \in \mathbf{R}^{d}\right)
$$

in place of $P_{n}(x)$. Namely

$$
B_{k}:=\left\{(n, x) \in \mathbf{N} \times \mathbf{Z}^{d}: p(n, x) \geq\left(2 \pi 2^{k}\right)^{-d / 2}|Q|^{-1 / 2}\right\} .
$$


We introduce also

$$
D_{p}:=\left\{(n, x) \in \mathbf{N} \times \mathbf{Z}^{d}:\|x\|^{2} \leq 2 p n\left(\log ^{+} \log n\right)\right\} .
$$

According to the law of the iterated logarithm the random walk $S_{n}$ eventually leaves the complement $\left[D_{p}\right]^{c}\left(:=\mathbf{N} \times \mathbf{Z}^{d} \backslash D_{p}\right)$ with probability one (see the Appendix). The next theorem is of more practical interest than the previous one.

Theorem 2. Let $A$ be a subset of $\mathbf{N} \times \mathbf{Z}^{d}$. Put $A_{k}:=A \cap\left(B_{k} \backslash B_{k-1}\right)$. Then

$$
P\{A \text { is hit i.o. }\}=1 \quad \text { if and only if } \sum_{k=1}^{\infty} h\left(\hat{0}, A_{k} \cap D_{p}\right)=\infty \text {; }
$$

moreover this is the case if and only if

$$
\sum_{k=1}^{\infty} 2^{-k d / 2} \operatorname{cap}\left(A_{k} \cap D_{p}\right)=\infty .
$$

Remark 1 . Theorem 1 (hence Corollary 1 as well) seems true quite generally (in the light of the fact that Wiener's test is valid for most space-time stable processes, as one can show along the same lines as in [U]); especially it would be enough to suppose $E\left\{\left|X_{1}\right|^{2}\right\}<\infty$ instead of (2.1) , but our proof needs the latter; Theorem 1 deduced from Theorem 2 with essential help of it. On the other hand Theorem 2 is not true under $E\left\{\left|X_{1}\right|^{2}\right\}<\infty$, so that Wiener's test must take a somewhat different form in the general case: this is because if it is assumed to the contrary to be true, then, as seen from the proof given in Section 7, Theorem 3 below must also be true (at least for $d=1$ ), but this contradicts a result of Feller [F2, Theorem 2] (see (iii) of Remark 4 in Section 7).

Theorem 2 (or Theorem 4 given in Section 6) provides simple proofs of well known tests such as Kolmogorov's and Dvoretzky-Erdös' (the continuous spacetime version of the former is also called Petrovskii's test). In particular, we shall obtain the following strengthening of the harder half of Kolmogorov's test . By our assumption of irreducibility we can find a minimal positive integer $\tau$ (the period) such that the whole space $\mathbf{Z}^{d}$ is divided into $\tau$ classes each of which is visited by the walk $S_{n}$ periodically (if $\tau \geq 2$ ), and $P_{n}(x)$ eventually becomes positive along a set of periodic times $\tau n+m$ of period $\tau$.

Theorem 3. Let $d=1$ and $\sigma^{2}=E\left\{X_{1}^{2}\right\}$ and $\tau$ be as above. Let $f(n)$ be an $\mathbf{N}$-valued function of $n \in \mathbf{N}$. Then $P\left\{S_{\tau n}=f(n)\right.$ for infinitely many $\left.n\right\}=1$ if

$$
\sum_{n=1}^{\infty} n^{-3 / 2} f(n) \exp \left(-\frac{f^{2}(n)}{2 n \tau \sigma^{2}}\right)=\infty
$$

A multidimensional analogue of Theorem 3 will be given in Section 7 .

Landis $[\mathrm{Ld}]$, studying a similar problem for the heat operator, divides the space by means of ordinate levels, which in our case amounts to using the infinite hyperslabs $\left(t_{k-1}, t_{k}\right] \times \mathbf{Z}^{d}$ in place of shells $B_{k}^{*} \backslash B_{k-1}^{*}$. However, this way never provides any test that is at least in the depth of Kolmogorov's. In fact the sequence $\left\{t_{k}\right\}$ cannot be finer than $\left\{a^{k}\right\}$ for any $a>1$ for the corresponding test (formulated like Theorem 1) to be valid (as a matter of fact $\left\{a^{k}\right\}$ is a proper choice in view of Theorem 4), but for getting Kolmogorov's test we must take them as fine as $\left\{e^{k / \log k}\right\}$ at least for typical f's (cf. [E]). 
In what follows we further assume, for simplicity, that the walk $S$ is strongly aperiodic, or equivalently, the period $\tau$ mentioned above is one. To assume this gives rise to no essential restriction since, otherwise, we may consider each of the walks $S_{\tau n}, S_{1+\tau n}, \ldots$, and $S_{\tau-1+\tau n}$ separately.

\section{A LOCAL Limit THEOREM}

We wish to compare $B_{k}^{*}$ and $B_{k}$. Specifically we wish to have that for some $N_{0} \in \mathbf{N}$

$$
B_{k-1} \cap D_{p, N_{0}} \subset B_{k}^{*} \cap D_{p, N_{0}} \subset B_{k+1} \cap D_{p, N_{0}},
$$

where $D_{p, N}:=\left\{(n, x) \in D_{p}: n \geq N\right\}$. This is an immediate consequence of the following proposition (see (3.3) below) for which we need our supplementary assumption of strong aperiodicity.

Proposition 1. Assume $S_{n}$ is strongly aperiodic. Then

$$
P_{n}(x)=p(n, x)+\frac{1}{n^{d / 2}(\log n)^{p}}\left(1 \wedge \frac{n}{|x|^{2}}\right) r(n, x),
$$

with $\lim _{n \rightarrow \infty} \sup _{x \in \mathbf{Z}^{d}} r(n, x)=0$, where $a \wedge b:=\min \{a, b\}$.

Corollary 2. Suppose $S_{n}$ is strongly aperiodic. Then for an $N_{0} \in \mathbf{N}$

$$
2^{-d / 2} p(n, x) \leq P_{n}(x) \leq 2^{d / 2} p(n, x) \quad \text { for } \quad(n, x) \in D_{p}, n \geq N_{0} .
$$

By summing up the right side of (3.2) over $\|x\| \leq \sqrt{n} g(n)$ and then subtracting the resultant from $1=\sum_{x \in \mathbf{Z}^{d}} p(n, x)+O(1 / \sqrt{n})$ we obtain the next corollary of Proposition 1, which is valid without assuming the strong aperiodicity.

Corollary 3. Let $g$ be a function on $\mathbf{N}$ with $g \geq 1$. Put

$$
v(n):= \begin{cases}1 & (d=1) \\ \log g(n) & (d=2), \\ g^{d-2}(n) & (d \geq 3) .\end{cases}
$$

Then there exists a constant $C$ such that

$$
P\left\{\left\|S_{n}\right\| \geq \sqrt{n} g(n)\right\} \leq C \max \left\{\frac{v(n)}{(\log n)^{p}}, \frac{1}{g(n)} \exp \left(-\frac{g^{2}(n)}{2}\right)\right\} .
$$

Proposition 1 would not be new. We indicate only the key steps of its proof. Let us write $X$ for $X_{1}$ and put

$$
\phi(\theta)=E\left\{e^{i \theta \cdot X}\right\} ; \quad Q(\theta)=E\left\{(\theta \cdot X)^{2}\right\} .
$$

Lemma 1. If $E X=0$ and $E\left\{|X|^{2}\left(\log ^{+}|X|\right)^{p}\right\}<\infty$ for some $p>0$, then as $\theta \rightarrow 0$

$$
\left|\nabla \phi(\theta)+\frac{1}{2} \nabla Q(\theta)\right| \leq E\left|\left(e^{i \theta \cdot X}-i \theta \cdot X\right) X\right|=o\left(|\theta| /|\log | \theta||^{p}\right)
$$

and

$$
\left.|\Delta \phi(\theta)+E| X\right|^{2} \mid \leq E\left\{\left|e^{i \theta \cdot X}-1\right||X|^{2}\right\}=o\left(1 /|\log | \theta||^{p}\right) .
$$


Proof of Lemma 1. For any positive number $\delta<1$

$$
\left|e^{i u}-\left(1+i u-\frac{1}{2} u^{2}\right)\right| \leq \begin{cases}\epsilon u^{2} /|\log | u||^{p} & (-\delta<u<\delta) \\ C u^{2} & (u \in \mathbf{R}),\end{cases}
$$

where $C$ is a constant independent of $\delta$ and $\epsilon=O\left(\delta|\log \delta|^{p}\right)=o(1)$ as $\delta \rightarrow 0$. Thus for $0<|\theta|<\delta^{2}$, which clearly implies $|X \cdot \theta| \leq \delta|\theta|^{1 / 2}|X|$,

$$
\begin{aligned}
\left|\phi(\theta)-1+\frac{1}{2} Q(\theta)\right| \leq & E\left\{\left|\exp (i \theta \cdot X)-\left(1+i \theta \cdot X-\frac{1}{2}(\theta \cdot X)^{2}\right)\right|\right\} \\
\leq & \epsilon E\left\{\frac{(\theta \cdot X)^{2}}{\left.|\log | \theta \cdot X\right|^{p}} ; 0<|X| \leq \frac{1}{|\theta|^{1 / 2}}\right\} \\
& +C E\left\{\frac{(\theta \cdot X)^{2}|\log | X||^{p}}{\left|\frac{1}{2} \log \right| \theta||^{p}} ;|X|>\frac{1}{|\theta|^{1 / 2}}\right\} .
\end{aligned}
$$

Since $\log |\theta \cdot X|^{-1} \geq \log |\theta|^{-1 / 2}$ for $|X| \leq 1 /|\theta|^{1 / 2}$, the right side above is dominated by

$$
2^{p} \frac{|\theta|^{2}}{\left.|\log | \theta\right|^{p}}\left[\epsilon E\left\{|X|^{2}\right\}+C E\left\{|X|^{2}|\log | X||^{p} ;|X|>\frac{1}{|\theta|^{1 / 2}}\right\}\right] .
$$

Thus the assumption of the lemma implies (3.4). The same argument proves the other asymptotic formulas.

Proof of Proposition 1. We show (3.2) for $|x|^{2} \geq n$. As in [S, Proposition 7.10], the proof is based on the following identities:

$$
\begin{aligned}
& \frac{|x|^{2}}{n}(2 \pi n)^{d / 2} P_{n}(x)=-\frac{1}{(2 \pi)^{d / 2}} \int_{\sqrt{n} T} e^{-i x \cdot \alpha / \sqrt{n}} \frac{1}{n}\left(\Delta \phi^{n}\right)\left(\frac{\alpha}{\sqrt{n}}\right) d \alpha, \\
& \frac{|x|^{2}}{n}(2 \pi n)^{d / 2} p(n, x)=-\frac{1}{(2 \pi)^{d / 2}} \int_{R^{d}} e^{-i x \cdot \alpha / \sqrt{n}} \Delta\left(e^{-\frac{1}{2} Q(\alpha)}\right) d \alpha,
\end{aligned}
$$

where $T=[-\pi, \pi]^{d}$. By (3.4) together with the relation $1-z=e^{-z}\left\{1+O\left(z^{2}\right)\right\}$ $(z \rightarrow 0)$ we first observe that

$$
\phi^{n}\left(\frac{\alpha}{\sqrt{n}}\right)=\exp \left\{-\frac{1}{2} Q(\alpha)\right\}\left\{1+o\left(\frac{|\alpha|^{2}}{(\log n)^{p}}\right)\right\}
$$

uniformly for $|\alpha| \leq(\log n)^{p / 2}$, and then, substituting (3.4) through (3.7) into the identity $\Delta \phi^{n}=n \phi^{n-1} \Delta \phi+n(n-1) \phi^{n-2}|\nabla \phi|^{2}$,

$$
\frac{1}{n} \Delta \phi^{n}\left(\frac{\alpha}{\sqrt{n}}\right)-\Delta\left(e^{-\frac{1}{2} Q(\alpha)}\right)=e^{-\frac{1}{2} Q(\alpha)} \times o\left(\frac{1+|\alpha|^{2}}{(\log n)^{p}}\right)
$$

with the same uniformity. On the other hand, we can choose $\delta>0$ small enough that

$$
\left|\frac{1}{n} \Delta \phi^{n}\left(\frac{\alpha}{\sqrt{n}}\right)\right| \leq \begin{cases}c e^{-\frac{1}{4} Q(\alpha)} & (|\alpha|<\delta \sqrt{n}), \\ c(1-\delta)^{n} & (\delta \sqrt{n}<|\alpha|, \alpha \in \sqrt{n} T)\end{cases}
$$


for some constant $c$, owing to the strong aperiodicity. Accordingly,

$$
\begin{aligned}
\frac{|x|^{2}}{n}(2 \pi n)^{d / 2}\left|P_{n}(x)-p(n, x)\right| \leq & \int_{R^{d}} e^{-\frac{1}{2} Q(\alpha)}\left(1+|\alpha|^{2}\right) d \alpha \times o\left(\frac{1}{(\log n)^{p}}\right) \\
& +c \int_{|\alpha|>(\log n)^{p / 2}} e^{-\frac{1}{4} Q(\alpha)} d \alpha+c(1-\delta)^{n}|\sqrt{n} T| \\
= & o\left(1 /(\log n)^{p}\right),
\end{aligned}
$$

uniformly in $x$ as desired. The proof of (3.2) for $|x|^{2}<n$ proceeds as in the standard manner with the help of (3.7) (cf. [S]).

\section{An INEQUaLity For hitTing PRobabilities}

For the proof of Theorems 1 and 2 the following proposition is fundamental. (In this section we have only to suppose the second moment condition $E\left\{|X|^{2}\right\}<\infty$ instead of (2.1) : specifically, we need the relation (3.3) only for $|x|^{2} \leq c n$ for each c.)

Proposition 2. There exist constants $\gamma<1, C>0$ and $n_{0} \in N$ such that if $\xi \in\left\{(n, x) \in B_{k}: n \geq n_{0}\right\}$ and $A \subset\left[B_{k+3}\right]^{c}$ for some $k$, then

$$
h(\xi, A) \leq \gamma+C h(\hat{0}, A) .
$$

For the proof of Proposition 2 (as well as for applications of Theorem 2) it is important to know the shape of $B_{k}$. (See Figure 1.) Put

$$
R_{k}(t):=\sqrt{-d t \log \left(t / 2^{k}\right)}, \quad 0 \leq t \leq 2^{k} .
$$

Then $(n, x) \in B_{k}$ if and only if $\|x\| \leq R_{k}(n)$. The continuum (the heat ball)

$$
\bar{B}_{k}:=\left\{(t, u) \in\left[0,2^{k}\right] \times \mathbf{R}^{d}:\|u\| \leq R_{k}(t)\right\}
$$

is an egg-shaped body with the top at $\left(2^{k}, \mathbf{0}\right)$ and its bottom (the center) at the origin $\hat{0}$, and broadest (relative to the norm $\|\cdot\|$ ) in the abscissa (i.e., space) direction at the ordinate level $t=2^{k} / e$ at which $R_{k}(t)=\sqrt{d t}$; its boundary surface intersects the parabolic surface $\|z\|^{2}=c t$ at the ordinate level $t=2^{k} e^{-c / d}$. It is worth noting, though not necessary for logically following the arguments given below, that the balls $\bar{B}_{k}$ and $\bar{B}_{k+m}$ are transformed to each other under the parabolic scaling: $(t, u) \leftrightarrow\left(2^{m} t, 2^{m / 2} u\right)$

We prepare two lemmas. The first one asserts that (4.1) is valid within each parabolic region:

Lemma 2. For any $c>0$ there exist constants $\gamma=\gamma_{c}<1, n_{c} \in \mathbf{N}$ and $C>0$ such that the inequality (4.1) holds if $\xi \in\left\{(n, x) \in B_{k}:\|x\|^{2} \leq c n, n \geq n_{c}\right\}$ and $A \subset\left[B_{k+3}\right]^{c}$.

Proof. Let $\xi=(n, x)$ with $\|x\|^{2} \leq c n$ and $\Lambda_{n}$ be the disk of the radius $n$ lying on the ordinate level $2 n$, i.e., $\Lambda_{n}:=\left\{(2 n, y):\|y\|^{2} \leq n\right\}$. We make use of the inequality

$$
\begin{aligned}
h(\xi, A) \leq 1 & -P\left\{\xi+\hat{S}_{j} \in B_{k+3} \quad \text { for } 1 \leq j<n \text { and } \xi+\hat{S}_{n} \in \Lambda_{n}\right\} \\
& +\sum_{(2 n, y) \in \Lambda_{n}} P\left\{x+S_{n}=y\right\} h((2 n, y), A) .
\end{aligned}
$$

Choose $n_{c}$ large enough that (3.3) is valid for $(n, y-x)$ (in place of $(n, x)$ ) whenever $(2 n, y) \in \Lambda_{n}$ and $n \geq n_{c}$. It is then clear that for some constant $C, P\left\{x+S_{n}=y\right\}$ 

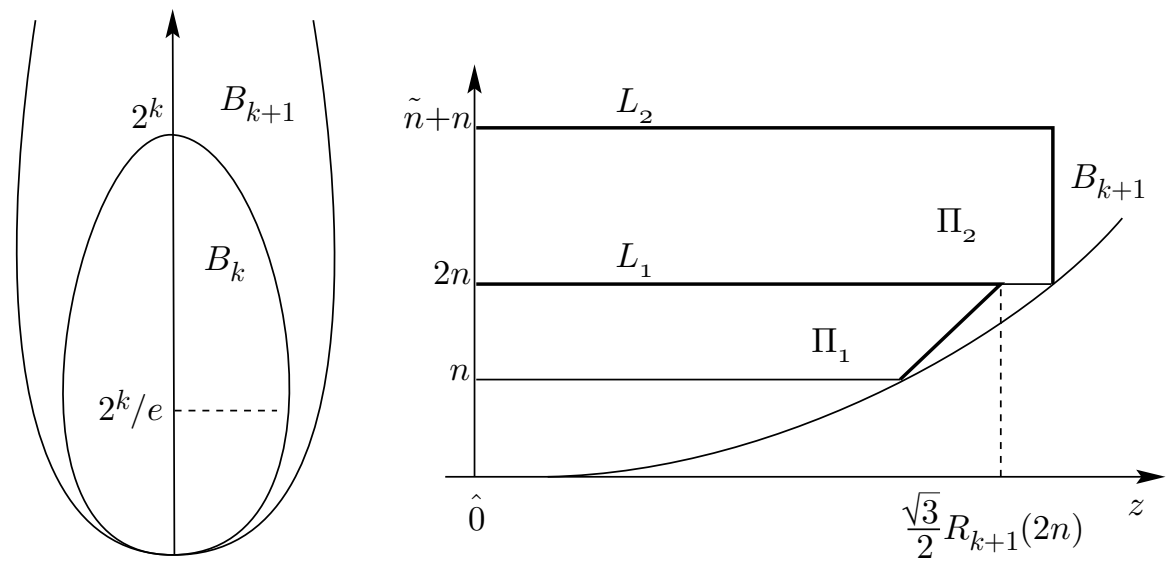

Figure 1

$\leq C P\left\{S_{2 n}=\right\}$ if $(2 n, y) \in \Lambda_{n}$ and $n \geq n_{c}$. Hence we can replace the last term on the right side in (4.3) by $C h(\hat{0}, A)$. It remains to show that for some $\gamma<1$

$$
P\left\{\xi+\hat{S}_{j} \in B_{k+3} \quad \text { for } \quad 1 \leq j \leq n \text { and } \xi+\hat{S}_{n} \in \Lambda_{n}\right\}>1-\gamma \quad\left(n \geq n_{c}\right) .
$$

Observe that $R_{k+1}(n)-\sqrt{c n} \geq[\sqrt{c+d \log 2}-\sqrt{c}] \sqrt{n}$ if $n \leq 2^{k} e^{-c / d}$, and so there exists a constant $\lambda=\lambda_{c}>0$ such that the ball $B_{k+3}$ contains the cylinder of lattice points $(m, z)$ determined by the inequalities $n \leq m \leq 2 n,\|z\|^{2} \leq(1+\lambda) n$. By using the invariance principle (if necessary) it then would be clear that (4.4) holds.

Lemma 3. For every $a>0$ there exist constants $b_{o}>0$ and $\alpha>0$ such that

$$
P\left\{\left\|S_{j}\right\| \leq a b^{-1} j+b \quad \text { for } j \geq 1\right\} \geq \alpha \quad \text { for } b \geq b_{o} .
$$

Proof. Assume $E\left\{\left\|X_{1}\right\|^{2}\right\}=1$ for simplicity. Define a sequence of integers $t_{k}$ ( $k=$ $0,1,2, \ldots)$ inductively by $t_{0}=0$ and $t_{k}-1<6(b k)^{2} / a^{2}+t_{k-1} \leq t_{k} \quad(k \geq 1)$, and consider the events $\mathcal{E}_{1}:=\left\{\left\|S_{j}\right\| \leq b\right.$ for $\left.1 \leq j \leq t_{1}\right\}$ and

$$
\mathcal{E}_{k}:=\left\{\left\|S_{j}-S_{t_{k-1}}\right\| \leq a b^{-1}\left(t_{k-1}-t_{k-2}\right) \quad \text { for } \quad t_{k-1}<j \leq t_{k}\right\} \quad(k \geq 2) .
$$

Then $\bigcap_{k=1}^{\infty} \mathcal{E}_{k} \subset\left\{\left\|S_{j}\right\| \leq(a / b) j+b\right.$ for $\left.j \geq 1\right\}$. By Kolmogorov's inequality,

$$
1-P\left\{\mathcal{E}_{k}\right\} \leq\left(k^{2}+a^{2} / 6 b^{2}\right) /\left(6(k-1)^{4}\right) \quad(k \geq 2) .
$$

Now, choosing a sufficiently large $b_{o}>a$ so that $P\left\{\mathcal{E}_{1}\right\}>0$ for $b \geq b_{o}$, we obtain $P\left\{\bigcap_{k=1}^{\infty} \mathcal{E}_{k}\right\}=\prod_{k \geq 1} P\left\{\mathcal{E}_{k}\right\} \geq \frac{1}{6} P\left\{\mathcal{E}_{1}\right\} \prod_{k \geq 3}\left[1-k^{2}(k-1)^{-4}\right]=: \alpha>0$. This finishes the proof of Lemma 3 .

Proof of Proposition 2. The proof is divided into two steps. 
Step 1. By virtue of Lemma 2 it suffices to prove that there is a constant $c(\geq d)$ such that if $\xi=(n, x)$ satisfies in addition $|x|^{2}>c n$, then (4.1) holds. Define

$$
\begin{aligned}
& \Pi_{1}=\left\{(n+j, z):\|z\| \leq \frac{\frac{\sqrt{3}}{2} R_{k+1}(2 n)-R_{k+1}(n)}{n} j+R_{k+1}(n), 0 \leq j \leq n\right\}, \\
& L_{1}=\left\{(2 n, z): \quad\|z\| \leq \frac{\sqrt{3}}{2} R_{k+1}(2 n)\right\}, \\
& \Pi_{2}=\left\{(n+j, z): \quad\|z\| \leq R_{k+1}(2 n), n \leq j \leq \tilde{n}\right\}, \\
& L_{2}=\left\{(n+\tilde{n}, z): \quad\|z\| \leq R_{k+1}(2 n)\right\}
\end{aligned}
$$

where

$$
\tilde{n}=n+\frac{1}{2}\left(1-\frac{\sqrt{3}}{2}\right)^{2} R_{k+1}^{2}(2 n) .
$$

Since the function $R_{k}(t)$ is concave and $\|z\| \leq R_{k}(n)$ implies $(n, z) \in B_{k}$, we have

$$
\Pi_{1} \subset B_{k+1} \text { and } L_{1} \subset B_{k+1} .
$$

(See Figure 1.) Let $\|x\|^{2} \geq c n$. From this and $(n, x) \in B_{k}$, we get $c n \leq R_{k}^{2}(n)$, or equivalently,

$$
n \leq 2^{k} \exp (-c / d)
$$

Noticing that $n+\tilde{n}=2 n\left[1+\frac{d}{2}\left(1-\frac{\sqrt{3}}{2}\right)^{2} \log \left(2^{k} / n\right)\right] \geq 2 n\left[1+\frac{1}{2}\left(1-\frac{\sqrt{3}}{2}\right)^{2} c\right]$, we observe that if $c$ is sufficiently large, then $R_{k+1}^{2}(2 n) \leq R_{k+1}^{2}(n+\tilde{n}) \wedge c(n+\tilde{n})$ and $n+\tilde{n} \leq 2^{k} / e$; hence

$$
\Pi_{2} \subset B_{k+1} \quad \text { and } \quad L_{2} \subset B_{k} \cap\left\{(n+\tilde{n}, z):\|z\|^{2} \leq c(n+\tilde{n})\right\},
$$

which we assume to hold below. By Lemma 2 there exist constants $\gamma_{c}<1, n_{c} \in \mathbf{N}$ and $C>0$ such that if $n+\tilde{n} \geq n_{c}$,

$$
h(\eta, A) \leq \gamma_{c}+C h(\hat{0}, A) \text { for } A \subset\left[B_{k+3}\right]^{c}, \eta \in L_{2} .
$$

If we define

$$
J_{\xi}=P\left\{\xi+\hat{S}_{j} \in B_{k+1} \quad \text { for } \quad 1 \leq j \leq \tilde{n} \quad \text { and } \quad \xi+\hat{S}_{\tilde{n}} \in L_{2}\right\},
$$

then

$$
\begin{aligned}
h(\xi, A) \leq & \sum_{\eta \in L_{2}} P\left\{\xi+\hat{S}_{j} \in B_{k+1} \text { for } 1 \leq j<\tilde{n} \text { and } \xi+\hat{S}_{\tilde{n}}=\eta\right\} h(\eta, A) \\
& \quad+1-P\left\{\xi+\hat{S}_{j} \in B_{k+1} \quad \text { for } 1 \leq j<\tilde{n} \text { and } \xi+\hat{S}_{\tilde{n}} \in L_{2}\right\} \\
\leq & J_{\xi} \sup _{\eta \in L_{2}} h(\eta, A)+1-J_{\xi} \\
\leq & 1-J_{\xi}\left(1-\gamma_{c}\right)+C J_{\xi} h(0, A) .
\end{aligned}
$$

Thus the proof of Proposition 2 will be complete, if we show there exists $c$ such that

$$
\inf \left\{J_{\xi}: \xi=(n, x) \in B_{k},\|x\|^{2}>c n\right\}>0 .
$$

This lower bound for $J_{\xi}$ is proved in the next step.

Step 2. The relation (4.8) is implied by the following two inequalities:

$$
\begin{gathered}
\inf _{(2 n, y) \in L_{1}} P\left\{(2 n, y)+\hat{S}_{j} \in \Pi_{2} \text { for } 1 \leq j \leq \tilde{n}-n\right\} \geq 1 / 2, \\
P\left\{\xi+\hat{S}_{j} \in \Pi_{1} \text { for } 1 \leq j \leq n\right\} \geq \alpha,
\end{gathered}
$$


where $\alpha>0$ is a constant depending only on $\left\{P_{n}(x)\right\}$ : in fact, by observing that

$$
J_{\xi} \geq P\left\{\xi+\hat{S}_{j} \in \Pi_{1} \quad \text { for } 1 \leq j \leq n ; \xi+\hat{S}_{j} \in \Pi_{2} \text { for } n \leq j \leq \tilde{n}\right\},
$$

and applying the Markov property, we deduce $J_{\xi} \geq \alpha / 2$ from (4.9) and (4.10). Since the event $\left\{(2 n, y)+\hat{S}_{j} \in \Pi_{2}\right.$ for $\left.1 \leq j \leq \tilde{n}-n\right\}$ includes

$$
\left\{\left\|S_{j}\right\| \leq\left(1-\frac{\sqrt{3}}{2}\right) R_{k+1}(2 n) \text { for } 1 \leq j \leq \tilde{n}-n\right\},
$$

inequality (4.9) is immediate from Kolmogorov's inequality.

It remains to prove that (4.10) is valid if $c$ is large enough. By observing that the relation (4.6) entails

$$
\left|R_{k+1}(2 n) / R_{k+1}(n)-\sqrt{2}\right| \leq \sqrt{2}[\sqrt{1+(d \log 2) / c}-1] \underset{c \rightarrow \infty}{\rightarrow} 0,
$$

we see that

$$
\begin{aligned}
n^{-1} & \left(\frac{\sqrt{3}}{2} R_{k+1}(2 n)-R_{k+1}(n)\right)\left(R_{k+1}(n)-R_{k}(n)\right) \\
& \geq \frac{\left(\frac{\sqrt{3}}{2} R_{k+1}(2 n)-R_{k+1}(n)\right)}{2 R_{k+1}(n)} d \log 2 \\
& \underset{c \rightarrow \infty}{\rightarrow} a_{o} \quad\left(a_{o}:=\frac{1}{2}\left(\sqrt{\frac{3}{2}}-1\right) d \log 2\right)
\end{aligned}
$$

uniformly in $(k, n)$ satisfying (4.6). Hence there exists a constant $c_{2}$ such that if $c \geq c_{2}$,

$$
n^{-1}\left(\frac{\sqrt{3}}{2} R_{k+1}(2 n)-R_{k+1}(n)\right) \geq \frac{1}{2} a_{o}\left(R_{k+1}(n)-R_{k}(n)\right)^{-1},
$$

so that the event $\left\{\xi+\hat{S}_{j} \in \Pi_{1}\right.$ for $\left.1 \leq i \leq n\right\}$ includes

$$
\left\{\left\|S_{j}\right\| \leq \frac{1}{2} a_{o}\left(R_{k+1}(n)-R_{k}(n)\right)^{-1} j+R_{k+1}(n)-R_{k}(n) \text { for } 1 \leq j \leq n\right\} .
$$

By Lemma 3 it is now clear that (4.10) holds. The proof of Proposition 2 is complete.

\section{Proof of Theorems 1 And 2}

We will apply the following version of the Borel-Cantelli lemma.

Lemma 4. Let $\mathcal{E}_{k}, k=1,2, \ldots$, be a sequence of events. Suppose there exist constants $\gamma<1$ and $C$ such that for positive integers $k$ and $M>k$

$$
P\left\{\mathcal{E}_{k} \cap\left(\bigcup_{m=k+1}^{M} \mathcal{E}_{m}\right)\right\} \leq\left[\gamma+C \sum_{m=k+1}^{M} P\left\{\mathcal{E}_{m}\right\}\right] P\left\{\mathcal{E}_{k}\right\} .
$$

Then $P\left\{\lim \sup _{k \rightarrow \infty} \mathcal{E}_{k}\right\}>0$ if and only if $\sum P\left\{\mathcal{E}_{k}\right\}=\infty$.

Proof. The idea of the proof is rather standard (cf. [E], [Lm]). Let $\sum P\left\{\mathcal{E}_{k}\right\}=\infty$. Suppose $\lim _{k \rightarrow \infty} P\left\{\mathcal{E}_{k}\right\}=0$, since otherwise the lemma is trivial. There then exists $L_{0}$ such that

$$
\begin{aligned}
& \text { for all } L \geq L_{0} \text { there exists } \quad M>L \text { such that } \\
& (1-\gamma) / 4 C \leq \sum_{k=L}^{M} P\left\{\mathcal{E}_{k}\right\} \leq(1-\gamma) / 2 C .
\end{aligned}
$$


Now by the second inequality and the assumption (5.1) we get the inequality $P\left\{\mathcal{E}_{k} \cap\left(\bigcup_{m=k+1}^{M} \mathcal{E}_{m}\right)\right\} \leq\left(\gamma+\frac{1}{2}(1-\gamma)\right) P\left\{\mathcal{E}_{k}\right\}$, or, rewriting it,

$$
P\left\{\mathcal{E}_{k} \backslash \bigcup_{m=k+1}^{M} \mathcal{E}_{m}\right\} \geq \frac{1}{2}(1-\gamma) P\left\{\mathcal{E}_{k}\right\} .
$$

The events $\mathcal{E}_{k} \backslash \bigcup_{m=k+1}^{M} \mathcal{E}_{m}, L \leq k<M$, and $\mathcal{E}_{M}$ are mutually disjoint with the union coinciding with that of the $\mathcal{E}_{k}$. Hence, the first inequality of (5.2), by summing up the right side in (5.3) over $k$ from $L$ through $M$, shows that $P\left\{\bigcup_{k=L}^{M} \mathcal{E}_{k}\right\}>$ $(1-\gamma)^{2} / 8 C$, so that $P\left\{\bigcap_{l=1}^{\infty} \bigcup_{k=l}^{\infty} \mathcal{E}_{k}\right\}=\lim _{L \rightarrow \infty} P\left\{\bigcup_{k \geq L} \mathcal{E}_{k}\right\}>0$.

Lemma 5. If $0 \leq \ell \leq k$ and $(n, x) \in B_{\ell}^{*}$, then

$$
P\left\{\hat{S}_{j} \notin B_{k}^{*} \text { for some } 1 \leq j<n ; S_{n}=x\right\} \leq 2^{-(k-\ell) d / 2} P_{n}(x) .
$$

Proof. We express the probability on the left side of (5.4) by means of the timereversed walk starting at $x$. Let us call it $S^{\bullet}$. Observing that $P\left(S_{n}^{\bullet}=0 \mid S_{0}^{\bullet}=x\right)=$ $P\left(S_{n}^{\bullet}=-x \mid S_{0}^{\bullet}=0\right)=P_{n}(x)$ and applying the strong Markov property, we see that the probability in (5.4) equals

$$
\begin{aligned}
P\left\{\hat{S}_{j}^{\bullet} \notin B_{k}^{*} \quad \text { for some } 1 \leq j<n \mid S_{n}^{\bullet}=0 ; S_{0}^{\bullet}=x\right\} P_{n}(x) \\
\quad=P\left\{\hat{S}_{j}^{\bullet} \notin B_{k}^{*} \quad \text { for some } 1 \leq j<n ; S_{n}^{\bullet}=0 \mid S_{0}^{\bullet}=x\right\} \\
\quad \leq \sup _{(m, y) \notin B_{k}^{*}} P\left\{S_{n}^{\bullet}=0 \mid S_{n-m}^{\bullet}=y\right\} \\
\quad=\sup _{(m, y) \notin B_{k}^{*}} P_{m}(y) .
\end{aligned}
$$

But $P_{m}(y) / P_{n}(x) \leq\left[2^{-k} / 2^{-\ell}\right]^{d / 2}=2^{-(k-\ell) d / 2}$ for $(n, x) \in B_{\ell}^{*}$ and $(m, y) \notin B_{k}^{*}$. Thus we obtain (5.4).

Proof of Theorem 2. By virtue of (2.3) and (3.1) it suffices to prove the first equivalence of Theorem 2. We can assume that $A \subset D_{p}$ in view of the law of the iterated logarithm. The proof of the 'only if' part then follows from the Borel-Cantelli lemma. For the proof of the converse part we put

$$
\mathcal{A}_{k}:=\bigcup_{n=1}^{\infty}\left\{\hat{S}_{j} \notin\left[B_{k+2}\right]^{c} \cap D_{p} \text { for } 1 \leq j<n ; \hat{S}_{n} \in A_{k}\right\},
$$

where $A_{k}:=A \cap\left(B_{k} \backslash B_{k-1}\right)$. We claim that for some constant $k_{0} \in \mathbf{N}$

$$
P\left\{\mathcal{A}_{k}\right\} \geq\left(1-2^{-d / 2}\right) h\left(\hat{0}, A_{k}\right) \quad \text { for } \quad k \geq k_{0} .
$$

For the proof of (5.5) we decompose the event $\mathcal{A}_{k}$ into subevents by means of the last leaving point, $(n, x)$ say, of the space-time process from $A_{k}$ to observe that

$$
\begin{gathered}
P\left(\mathcal{A}_{k}\right) \geq \sum_{(n, x) \in A_{k}} P\left\{\hat{S}_{j} \notin\left[B_{k+2}\right]^{c} \cap D_{p} \text { for } 1 \leq j<n ; S_{n}=x\right\} \\
\times P\left\{(n, x)+\hat{S}_{j} \notin A_{k} \text { for } j \geq 1\right\} .
\end{gathered}
$$

The sets $\left[B_{k-1}\right]^{c} \cap D_{p}$ get separated from the zero-th level $\{0\} \times \mathbf{Z}^{d}$ unrestrictedly, as $k$ becomes indefinitely large, so that the relation (3.3) is applicable for points of $\left[B_{k+2}\right]^{c} \cap D_{p}$ and for $(n, x) \in A_{k}$. Taking this into account we apply Lemma 5 to see that the first factor under the sum on the right side of (5.6) is at least 
$\left(1-2^{-d / 2}\right) P_{n}(x)$. Carrying out the summation with this bound we obtain the claimed inequality (5.5).

The rest of the proof is easy. Assume $\sum_{k=1}^{\infty} h\left(\hat{0}, A_{k}\right)=\infty$. Then, in view of (5.5), $\sum_{k=1}^{\infty} P\left\{\mathcal{A}_{k}\right\}=\infty$. Choose a positive integer $j \in\{0,1,2,3\}$ such that $\sum_{k=1}^{\infty} P\left\{\mathcal{A}_{4 k+j}\right\}=\infty$. We can assume $j=0$ without loss of generality, the other cases of $j$ being dealt with in the same way. Thus, it is sufficient to show that $P\left\{\bigcap_{l=1}^{\infty} \bigcup_{k=l}^{\infty} \mathcal{A}_{4 k}\right\}>0$ in view of the Hewitt-Savage zero-one law. Recall the occurrence of $\mathcal{A}_{4 k}$ implies that the sets $A_{4 m}, m>k$, cannot be hit before $A_{4 k}$ is hit. Then applying the strong Markov property and Proposition 2, we get

$$
\begin{aligned}
P\left\{\left(\bigcup_{m=k+1}^{M} \mathcal{A}_{4 m}\right) \cap \mathcal{A}_{4 k}\right\} & \leq P\left\{\mathcal{A}_{4 k}\right\}\left[\max _{\xi \in A_{4 k}} h\left(\xi, \bigcup_{m=k+1}^{M} A_{4 m}\right)\right] \\
& \leq P\left\{\mathcal{A}_{4 k}\right\}\left[\gamma+C \sum_{m=k+1}^{M} h\left(\hat{0}, A_{4 m}\right)\right] .
\end{aligned}
$$

The inequality (5.1) being satisfied for $\mathcal{E}_{k}=\mathcal{A}_{4 k}$ in view of (5.5) again, an application of Lemma 4 completes the proof of Theorem 2.

Proof of Theorem 1. With (3.1) taken into account Theorem 2 immediately follows from Theorem 2 and the following

\section{Lemma 6.}

$$
\sum_{k=1}^{\infty} h\left(\hat{0},\left[B_{k}^{*} \backslash B_{k-1}^{*}\right] \cap\left[D_{p}\right]^{c}\right)<\infty .
$$

Proof. As an approximate solution to the equation $R_{k+2}(t)=\sqrt{2 p t \log \log t}$, namely $2^{k+2}=t(\log t)^{2 p / d}$, we adopt the integer

$$
n=n_{k}:=\left\lfloor 2^{k+2}((k+2) \log 2)^{-2 p / d}\right\rfloor
$$

$(\lfloor u\rfloor$ denotes the largest integer that does not exceed $u$ ). Then for $k$ large enough

$$
\begin{aligned}
& h\left(\hat{0},\left[B_{k+1}^{*} \backslash B_{k}^{*}\right] \cap\left[D_{p}\right]^{c}\right) \\
& \leq P\left\{\left\|S_{n}\right\| \geq \sqrt{2 p n \log \log n}\right\} \\
& \quad+\sum_{\ell=1}^{k+3} P\left\{\hat{S}_{j} \notin B_{k}^{*} \text { for some } j \leq n ; \hat{S}_{n} \in B_{\ell}^{*} \backslash B_{\ell-1}^{*}\right\} .
\end{aligned}
$$

An application of Corollary 3 in Section 3 shows that the first term on the right side is at most a constant multiple of $(\log n)^{-p}(\log \log n)^{d / 2} \asymp k^{-p}(\log k)^{d / 2}$, where $a_{k} \asymp b_{k}$ means the ratio $a_{k} / b_{k}$ is bounded off zero and infinity. On the other hand Lemma 5 tells us that the second term is dominated by

$$
\begin{aligned}
\sum_{\ell=1}^{k+3} \sum_{x:(n, x) \in B_{\ell}^{*} \backslash B_{\ell-1}^{*}} P_{n}(x) 2^{\ell d / 2} 2^{-k d / 2} & \leq 2^{k d / 2} \sum_{\ell=1}^{k+3} \sum_{x:(n, x) \in B_{\ell}^{*} \backslash B_{\ell-1}^{*}} 1 \\
& \asymp 2^{-k d / 2}\left[R_{k}(n)\right]^{d} \asymp k^{-p}(\log k)^{d / 2} .
\end{aligned}
$$

Accordingly the series in the lemma converges.

Remark 2. If in Theorem 2 the set $A$ is included in a parabolic region $\|x\|^{2} \leq$ $c n$ as in the Dvoretsky-Erdös test ( $c$ may be any constant), we do not need the full strength of Proposition 2: Lemma 2 is enough [in the proof of Theorem 2, 
Proposition 2 is applied only for the second inequality of (5.7)]. (By the way, the test is vaild under $E\left\{\left|X_{1}\right|^{2}\right\}<\infty$ for such an $A$.) The gap between Lemma 2 and Proposition 2 may be explained by a singular behaviour of the Green function $P_{n}(x)$ when $\|x\|^{2} / n$ gets large (or by that of $R_{k}(n)$ for small $n / 2^{k}$ ) and is reflected by the fact that any proof of Kolmogorov's test available in the literature is far more involved than the proof of the Dvoretsky-Erdös test.

Remark 3. One of the main tools in [EG] is an extension of Harnack's inequality for the heat equation (Lemma 3.2). Its random walk analogue may read (in terms of harmonic measures) as follows: If for $\xi=(n, x)$ and $\eta=(m, y)$ we set

$$
q_{k}(\xi, \eta)=P\left\{\xi+\hat{S}_{j} \in B_{k+1} \text { for } 1 \leq j \leq m-n ; \xi+\hat{S}_{m-n}=\eta\right\},
$$

then for any $0<\delta<1$ there exists a constant $\kappa(\delta)>0$ such that

$$
\inf _{\xi \in B_{k}} \inf _{\|y\| \leq \delta R_{k+1}(N)} q_{k}(\xi,(N, y)) \geq \frac{\kappa(\delta)}{\sqrt{N^{d}}}, \quad \text { where } \quad N=\frac{3}{2} 2^{k},
$$

for all sufficiently large $k$. This bound of $q_{k}$ implies Proposition 2 as we can easily see. Conversely, our lower bound (4.8) for $J_{\xi}$ verifies (5.8), provided that the inequality in (5.8) is true if the range of the first infimum is further restricted to the parabolic regions, namely: for every $c>1$

$$
\inf _{\xi \in B_{k}:\|x\|^{2}<c n} \inf _{\|y\| \leq \delta R_{k+1}(N)} q_{k}(\xi,(N, y)) \geq \frac{\kappa(\delta, c)}{\sqrt{N^{d}}} .
$$

The bound (5.9) follows from the corresponding bound for $q_{k}^{*}$ that is defined with $B_{k+1}^{*}$ in place of $B_{k+1}$ (at least under (2.1)); the latter can be readily proved by making use of the relation $q_{k}^{*}(\xi,(N, y))=\sum_{z} q_{k}^{*}(\xi,(2 n, z)) q_{k}^{*}((2 n, z),(N, y)$ ) (with $\left.\xi=(n, x) \in B_{k}^{*},\|x\|^{2}<c n\right)$ and by substituting (5.4) into the same relation but with $\xi=\hat{0}$ to obtain $\sum_{z:\|z\|^{2}<c^{\prime} n} q_{k}^{*}((2 n, z),(N, y))>\kappa^{\prime} / \sqrt{N^{d}}$ (with $c^{\prime}$ large enough and $\left.n<\frac{1}{100} N\right)$.

\section{REFINEMENT}

For each $k$ define a sequence of positive numbers $\left\{T_{j}^{k}\right\}_{j=-1}^{\infty}$ by

$$
T_{-1}^{k}:=2^{k}, \quad T_{0}^{k}:=2^{k-1} / e
$$

for the first two entries and then inductively by

$$
R_{k}\left(T_{j+1}^{k}\right)=R_{k-1}\left(T_{j}^{k}\right), \quad T_{j+1}^{k} \leq 2^{k-1} / e .
$$

By means of this sequence, we define a partition $\left\{U_{j}^{k}\right\}$ of $B_{k} \backslash B_{k-1}$ by

$$
U_{j}^{k}:=\left\{(n, x) \in B_{k} \backslash B_{k-1}: T_{j+1}^{k}<n \leq T_{j}^{k}\right\} .
$$

(See Figure 2.) The importance of this partition consists in its geometry compatible with the parabolic scaling, i.e., the height of the $j$-th unit $U_{j}^{k}$ is in the same order as the square of its width with the asymptotic order getting precise as $j \rightarrow \infty$. This fact is seen by observing that

$$
\begin{aligned}
T_{j}^{k}-T_{j+1}^{k} & =T_{j}^{k}\left[1-\frac{\log \left(T_{j}^{k} / 2^{k-1}\right)}{\log \left(T_{j+1}^{k} / 2^{k}\right)}\right]=T_{j}^{k} \frac{\log \left(2 T_{j}^{k} / T_{j+1}^{k}\right)}{-\log \left(T_{j+1}^{k} / 2^{k}\right)} \\
& \sim \frac{T_{j}^{k} \log 2}{-\log \left(T_{j}^{k} / 2^{k}\right)}=(d \log 2)\left[\frac{T_{j}^{k}}{R_{k}\left(T_{j}^{k}\right)}\right]^{2}
\end{aligned}
$$



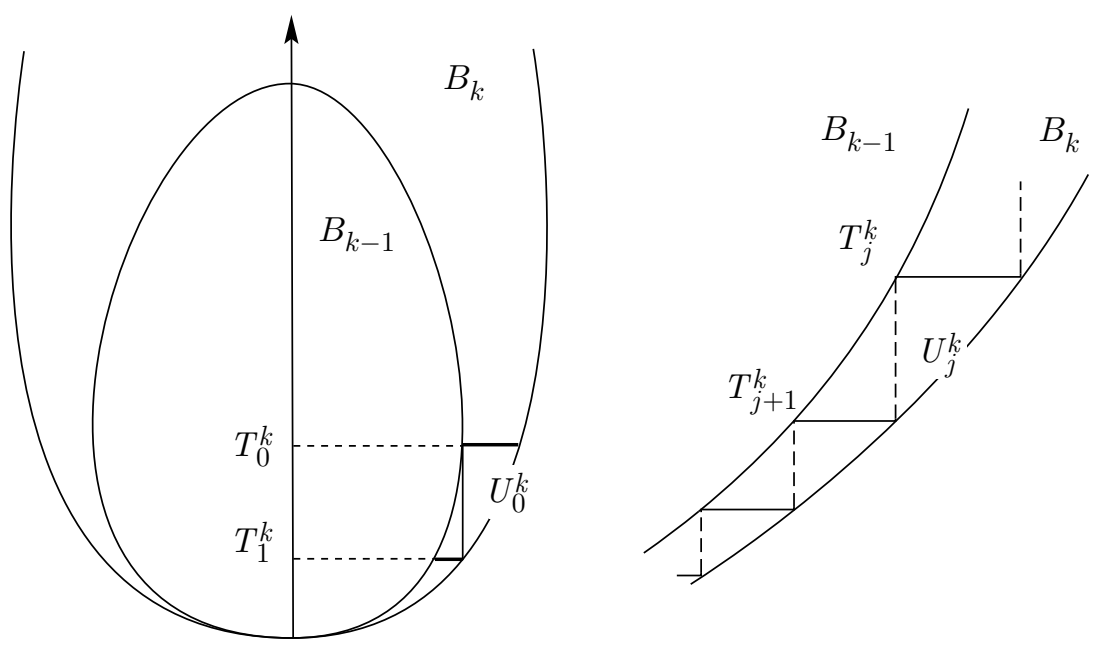

Figure 2

together with $T_{j}^{k} / T_{j+1}^{k} \rightarrow 1$, and then

$$
R_{k}\left(T_{j}^{k}\right)-R_{k-1}\left(T_{j}^{k}\right)=\frac{(d \log 2) T_{j}^{k}}{R_{k}\left(T_{j}^{k}\right)+R_{k-1}\left(T_{j}^{k}\right)} \sim \frac{1}{2} \sqrt{d \log 2} \sqrt{T_{j}^{k}-T_{j+1}^{k}},
$$

uniformly in $k$ as $j \rightarrow \infty$, where $a_{j} \sim b_{j}$ means $\lim a_{j} / b_{j}=1$. Note that for each $j$ the units $U_{j}^{k}, k=1,2, \ldots$, are transformed into one another under parabolic scaling, in particular the ratios $T_{j}^{k} / T_{j+1}^{k}(\geq 1)$ do not depend on $k$ and hence are bounded above.

Theorem 4. The condition (2.4) in Theorem 2, i.e., $\sum 2^{-k d / 2} \operatorname{cap}\left(A_{k} \cap D_{p}\right)=\infty$, is equivalent to the following condition:

$$
\sum_{k=1}^{\infty} 2^{-k d / 2} \sum_{j=-1}^{\infty} \operatorname{cap}\left(A \cap U_{j}^{k} \cap D_{p}\right)=\infty .
$$

Proof of Theorem 4. Clearly (2.4) follows from (6.3). The converse assertion follows from (2.3), (3.1) and the following

Lemma 7. Set $A_{k, j}:=A \cap U_{j}^{k} \cap D_{p}$. Assume limsup $\sup _{k \rightarrow \infty} h\left(\hat{0}, A_{k} \cap D_{p}\right)=0$. Then there exist $k_{0} \in N$ and $C$ such that

$$
\sum_{j=-1}^{\infty} h\left(\hat{0}, A_{k, j}\right) \leq C h\left(\hat{0}, A_{k} \cap D_{p}\right) \quad \text { for } \quad k \geq k_{0} .
$$

Proof. We may assume $\sum_{j=0}^{\infty} h\left(\hat{0}, A_{k, 2 j}\right) \leq \sum_{j=-1}^{\infty} h\left(\hat{0}, A_{k, 2 j+1}\right)$, being able to deal with the other case in the same way. By dividing the event that the set $\bigcup_{j=-1}^{\infty} A_{k, 2 j+1}$ is hit into disjoint events according as which of the $A_{k, 2 j+1}$ is hit last, we get

$$
\sum_{i=0}^{\infty}\left\{1-\sup _{\xi \in A_{k, 2 i+1}} h\left(\xi, \bigcup_{j=-1}^{i-1} A_{k, 2 j+1}\right)\right\} h\left(\hat{0}, A_{k, 2 i+1}\right) \leq h\left(\hat{0}, A_{k} \cap D_{p}\right) .
$$


Therefore it suffices to prove that there exist constants $q>0$ and $k_{0} \in \mathbf{N}$ such that

$$
1-\sup _{\xi \in A_{k, 2 i+1}} h\left(\xi, \bigcup_{j=-1}^{2 i-1} A_{k, j}\right) \geq q \text { for } k \geq k_{0} .
$$

Let $t_{k}$ be the ordinate at which $B_{k+1}$ intersects $D_{p}$, and $B^{\prime}(k-4)$ the intersection of $B_{k-4}$ with the upper half space $\left[t_{k-1}, \infty\right) \times \mathbf{Z}^{d}$. Then $1-h\left((n, x), \bigcup_{j=-1}^{2 i-1} A_{k, j}\right)$ is bounded below by

$$
P\left\{(n, x)+\hat{S}_{\left\lfloor T_{2 i}^{k}\right\rfloor-n} \in B_{k-4}\right\}\left(1-\sup _{\eta \in B^{\prime}(k-4)} h\left(\eta, \bigcup_{j=-1}^{2 i-1} A_{k, j}\right)\right)
$$

if $(n, x) \in A_{k, 2 i+1}$ (entailing $t_{k-1} \leq n \leq T_{2 i+1}^{k}$ ). By Proposition 2 the second factor is bounded below by $1-\gamma-C h\left(\hat{0}, A_{k} \cap D_{p}\right)$, which converges to $1-\gamma$ as $k \rightarrow \infty$. An application of the central limit theorem with the help of (6.2) verifies that the first factor is also bounded below by a positive constant. Thus Lemma 7 has been proved.

In the case when $d \geq 2$ we can further partition each set $U_{j}^{k}$ according to a partition of the unit sphere $\Theta$. As is realized from the proof of Lemma 7, such partitioning does no harm to the test as long as the bottom ends of the resulting pieces, $U_{j, i}^{k}$ say, have diameters of the same order as the thickness of the ring $U_{j}^{k}$ $\left(\sim\left[R_{k}\left(T_{j}^{k}\right)-R_{k-1}\left(T_{j}^{k}\right)\right]\right)$ so that the following condition is fulfilled:

$$
\left\{\begin{array}{l}
\text { there exist constants } \epsilon>0 \text { and } \nu=\nu_{d} \in \mathbf{N} \text { such that for each } k \text { and } \\
j \geq 1 \text { the family }\left\{U_{j, i}^{k}\right\}_{i} \text { is divided into } \nu \text { subfamilies so that } \\
\text { members within the same subfamily keep distance from one another } \\
\text { by more than } \epsilon\left[R_{k}\left(T_{j}^{k}\right)-R_{k-1}\left(T_{j}^{k}\right)\right] .
\end{array}\right.
$$

In fact, from $(\star)$ and $(6.2)$ we deduce $\sum_{i} h\left(\hat{0}, U_{j, i}^{k}\right) \leq \operatorname{const} . h\left(\hat{0}, U_{j}^{k}\right)$, hence the following:

Theorem 5. Let $d \geq 2$, and let $U_{j}^{k}$ be further partitioned into blocks $U_{j, i}^{k}$ for which the condition $(\star)$ above holds. Then the condition (2.4) is equivalent to

$$
\sum_{k=1}^{\infty} 2^{-k d / 2} \sum_{j=-1}^{\infty} \sum_{i} \operatorname{cap}\left(A \cap U_{j, i}^{k} \cap D_{p}\right)=\infty .
$$

\section{Applications}

We continue to assume that the walk $S_{n}$ is strongly aperiodic; all the results in this section are valid without this restriction if they are modified as in Theorem 3.

7.1. Let $f(n)$ be a positive function of $n \in \mathbf{N}$. Put $h(n)=f(n) / \sqrt{n}$. Kolmogorov's test states that if $h(n)$ is non-decreasing, then

$$
P\left\{\left\|S_{n}\right\| \geq f(n) \text { for infinitely many } n\right\}=0 \text { or } 1
$$

according as the series

$$
I\{h\}:=\sum_{n=1}^{\infty} \frac{1}{n} h^{d}(n) \exp \left(-\frac{1}{2} h^{2}(n)\right)
$$


converges or diverges. We treat the two halves of Kolmogorov's test separately, our main interest being in the harder half of it, as it should be. The following theorem improves this half in two ways, i.e., the monotonicity assumption on $h$ is removed and the set to test is reduced: it is now the graph of $f$ rather than the outer region marked off by $f$.

Theorem 6. If $d \geq 5$, further assume $E\left\{\left|X_{1}\right|^{3}\right\}<\infty$. Put

$$
F:=\left\{(n, x) \in \mathbf{N} \times \mathbf{Z}^{d}: f(n) \leq\|x\| \leq f(n)+\delta_{o}\right\} .
$$

Here $\delta_{o}$ is a constant that is not too small; one may take $\delta_{o}=\sqrt{\sum_{1 \leq i, j \leq d}\left(Q^{-1}\right)_{i, j}}$ to be definite. Then $P\{F$ is hit i.o. $\}=1$ if $I\{h\}=\infty$.

Proof. We apply Theorem 5 with the help of Lemma 8 of the next section. The latter says that there exists a positive constant $C$ such that for $j \geq 0$ and $k \geq 2$

$$
\operatorname{cap}\left(F \cap U_{j, i}^{k} \cap D_{p}\right) \geq \frac{C}{\sqrt{T_{j}^{k}-T_{j+1}^{k}}} \sharp\left(F \cap U_{j, i}^{k} \cap D_{p}\right),
$$

where $U_{j, i}^{k}$ and $T_{j}^{k}$ are introduced in the previous section and $\sharp$ indicates cardinality of a set. ( $U_{j, i}^{k}$ is understood to be $U_{j}^{k}$ for all $i$ when $d=1$.) We are going to show that there exists $C^{\prime}$ such that

$$
\sum_{n \in H} \frac{1}{n} h^{d}(n) \exp \left(-\frac{1}{2} h^{2}(n)\right) \leq C^{\prime} \sum_{k} 2^{-k d / 2} \sum_{j} \sum_{i} \frac{\sharp\left(F \cap U_{j, i}^{k} \cap D_{p}\right)}{\sqrt{T_{j}^{k}-T_{j+1}^{k}}},
$$

where $H:=\left\{n \in \mathbf{N}: f(n)+\delta_{o} \leq \sqrt{2 p n \log \log n}\right\}$. Theorem 5 combined with (7.2) and (7.3) readily verifies the assertion of the theorem.

Let us show (7.3). Suppose $n \in H$ and $\left[F \cap U_{j, i}^{k}\right] \cap\left[\{n\} \times \mathbf{Z}^{d}\right] \neq \emptyset$. Then, on the one hand, by the definition of $B_{k}$

$$
p(n, f(n))=(2 \pi n)^{-d / 2} \exp \left(-\frac{1}{2} h^{2}(n)\right) \leq C_{1} 2^{-k d / 2}
$$

for some constant $C_{1}$. On the other hand, employing (6.1), we see that

$$
T_{j}^{k}-T_{j+1}^{k} \asymp\left[T_{j}^{k} / R_{k}\left(T_{j}^{k}\right)\right]^{2}
$$

( $\asymp$ indicates that the ratio of the two sides of it is bounded off from both 0 and infinity) as well as $f(n) \leq C_{2} R_{k}\left(T_{j}^{k}\right)$ and $n \asymp T_{j}^{k}$, to obtain

$$
f(n) / n \leq C_{3} / \sqrt{T_{j}^{k}-T_{j+1}^{k}} .
$$

Finally, $\sharp\left(F \cap\left[\{n\} \times \mathbf{Z}^{d}\right]\right) \asymp f^{d-1}(n)$. From this together with (7.4) and (7.5) we get (7.3). Thus the proof of Theorem 6 is finished.

When $d \geq 2$ we can generalize Theorem 6 by allowing the function $f$ to depend on the space variable $x$ through its direction. Let $f(n, \theta)$ be a positive function of $n \in \mathbf{N}$ and $\theta \in \Theta$, where $\Theta$ is the $(d-1)$-dimensional unit sphere. Put

$$
F:=\left\{(n, x) \in \mathbf{N} \times \mathbf{Z}^{d}: f(n, x /|x|) \leq\|x\| \leq f(n, x /|x|)+\delta_{o}\right\} .
$$

Here $\delta_{o}$ is the same constant as in Theorem 6 . By slightly modifying the proof of Theorem 6 we can verify 
Theorem 7. Let $d \geq 2$. Suppose for each $n$ the function $f(n, \cdot)$ is piecewise constant in such a way that each region of constancy is convex (on the sphere) and its radius is greater than $1 / f(n, \theta)$, where $\theta$ is any point of the region. If $d \geq 5$, further assume $E\left\{\left|X_{1}\right|^{3}\right\}<\infty$. Then for $P\{F$ is hit $i . o\}=$.1 it is sufficient that

$$
\sum_{n=1}^{\infty} n^{-1-d / 2} \int_{\Theta} f^{d}(n, \theta) \exp \left(-\frac{f^{2}(n, \theta)}{2 n}\right) d \theta=\infty
$$

The converse of Theorem 6 cannot be claimed unless some regularity condition is imposed on the function $f$. In Kolmogorov's test $h$ is supposed to be monotone. This restriction is relaxed to either of the following conditions

$$
\begin{gathered}
\inf _{n \in \mathbf{N}} \inf _{0<m \leq n / h^{2}(n)}\left[h^{2}(n+m)-h^{2}(n)\right]>-\infty, \\
\sup _{n \in \mathbf{N}} \sup _{0<m \leq n / h^{2}(n)} \max \left\{\left[h^{2}(n+m)-h^{2}(n)\right],[h(n) / h(n+m)]\right\}<\infty .
\end{gathered}
$$

Theorem 8. Suppose that either (7.6a) or (7.6b) holds and that $\liminf h(n)>0$. Then $P\left\{\left\|S_{n}\right\|<f(n)\right.$ except for a finite number of $\left.n\right\}=1$ if $I\{h\}<\infty$.

The proof of Theorem 8 is relegated to the Appendix. Here we note that condition (7.6a) is satisfied for every non-decreasing $f$. In fact we have the following

Lemma 8. The condition (7.6a) is satisfied if $\inf _{t>1} t f^{\prime}(t) / f(t)>-\infty$ for a suitable smooth extension of $f$ to the positive reals, or equivalently,

$$
\inf _{t>1} t h^{\prime}(t) / h(t)>-\infty .
$$

Proof. The two conditions in the lemma are equivalent simply because $f^{\prime}(t) / f(t)$ $=(2 t)^{-1}+h^{\prime}(t) / h(t)$. Given $t>0$, we let the function $h(t+s), 0 \leq s \leq t / h^{2}(t)$, attain its minimum at a point $b$ and put $a=\max \{s \leq b: h(t+s)=h(t)\}$. Then, for some $\theta$ from the interval $[t+a, t+b]$,

$$
\inf _{0<s<t / h^{2}(t)}\left[h^{2}(t+s)-h^{2}(t)\right]=h^{2}(t+b)-h^{2}(t+a)=2 h(\theta) h^{\prime}(\theta)(b-a) .
$$

Since $b-a \leq t / h^{2}(t) \leq \theta / h^{2}(\theta)$ and $h^{\prime}(\theta) \leq 0$, the last member is bounded below by $2 \theta h^{\prime}(\theta) / h(\theta)$. Thus (7.6a) holds if (7.7) does.

Remark 4. (i) Kolmogorov's test is stated, without proof, in Lévy's book [Lv, p. 266]. It is proved by Erdös [E] for the simple random walk on $\mathbf{Z}$. The continuous space-time analogue is known as Petrovskii's test among analysts, having been proved by Petrovskii $[\mathrm{P}]$ as a test for a regular point for the heat operator; an elegant probabilistic proof of it is given by Motoo [M].

(ii) W. Feller extended Erdös' result significantly in two ways: firstly to sums of independent, but not necessarily identically distributed, $\mathbf{R}$-valued random variables subject to certain growth conditions (including the special case of identical distribution with the moment condition (2.1)) in [F1], and secondly to sums of $\mathbf{R}$-valued i.i.d. variables having the second moment in [F2].

(iii) In [F2] it is shown, in particular, that Kolmogorov's test is true (resp. false) if $(\log \log x) E\left\{X_{1}^{2} ;\left|X_{1}\right|>x\right\}$ is bounded (resp. diverges to infinity) as $x \rightarrow$ $\infty$. The manner in which the longer tail of the distribution of $X_{1}$ invalidates the criterion given by the infinite sum $I\{h\}$ is to make the class of upper functions larger, implying that if $(\log \log x) E\left\{X_{1}^{2} ;\left|X_{1}\right|>x\right\} \rightarrow+\infty$ then there exists an increasing $h$ that belongs to the upper class and for which $I\{h\}=\infty$. 
7.2. Let $f(n)=h(n) \sqrt{n}>0$ as before. The Dvoretzky-Erdös test states that if $d \geq$ 3 and $h(n)$ is non-increasing, then $P\left\{\left\|S_{n}\right\|<f(n)\right.$ for infinitely many $\left.n\right\}=0$ or 1 according as $\sum_{k=1}^{\infty} h^{d-2}\left(2^{k}\right)$ converges or diverges. This result follows from Theorem 2 and Lemma 9 in the next section, the latter showing that the capacity of the set $\left\{(n, x): 2^{k} \leq n<2^{k+1},\|x\|<f(n)\right\}$ is bounded above by a constant multiple of $2^{k} f^{d-2}\left(2^{k}\right)=2^{k d / 2} h^{d-2}\left(2^{k}\right)$ and bounded below by const. $2^{k d / 2} h^{d-2}\left(2^{k+1}\right)$.

7.3. Let $d=2$. The time axis then being hit i.o., we consider a test for the sets in the form $A=\{(t(m), \mathbf{0}): m \in \mathbf{N}\}$, where $\{t(m)\}$ is any ascending sequence of positive integers. The situation is very similar to the criterion for whether the set of points $(t(m), 0,0) \in \mathbf{Z}^{3}$ is hit infinitely many times by a 3 -dimensional random walk having mean zero and finite variance. In view of Theorem 2 a result of [IM] implies that if $t(m+1)-t(m) \geq \delta \log t(m)$ for some constant $\delta>0$, then $P\left\{S_{t(m)}=\mathbf{0}\right.$ for infinitely many $\left.m\right\}=0$ or 1 according as $\sum_{m=1}^{\infty} 1 / t(m)$ converges or diverges.

\section{Evaluation OF CAPACity}

We collect in this section certain estimates, from below or above, of capacities of sets having various shapes. These are not only applied in this paper, but also interesting for their own sake. The tool we employ for the proof is exclusively the variational characterization of capacity that may read as follows: Let $A$ be a bounded set of $\mathbf{Z}^{d+1}, \mu$ a non-negative measure on $A$ and $c$ a positive constant. Then

$$
\operatorname{cap}(A) \leq c^{-1} \sum_{\eta \in A} \mu(\eta) \quad \text { if } \quad \sum_{\eta \in A} G(\xi, \eta) \mu(\eta) \geq c \quad \text { for every } \quad \xi \in A ;
$$

similarly $\operatorname{cap}(A) \geq c^{-1} \sum_{\eta \in A} \mu(\eta)$ if $\sum_{\eta \in A} G(\xi, \eta) \mu(\eta) \leq c$ for every $\xi \in A$.

Lemma 8. If $d \geq 5$, further assume $E\left\{\left|X_{1}\right|^{3}\right\}<\infty$. Let $\delta_{o}=\sqrt{\sum\left(Q^{-1}\right)_{i, j}}$. If $N \in \mathbf{N}$ and the set $A$ satisfies

$$
A \subset\left\{(n, x): 0 \leq n \leq N ; f(n) \leq\|x\| \leq f(n)+\delta_{o} ;\left|x^{(2)}\right|+\cdots+\left|x^{d}\right| \leq C \sqrt{N}\right\}
$$

for some positive function $f$ and some non-negative constant $C$, where $x^{(i)}$ indicates the $i$-th component of $x$, then $\operatorname{cap}(A) \geq K(\sharp A) / \sqrt{N}$, where $K$ is a constant depending only on $C$ and $\left\{P_{1}(x)\right\}$.

Proof. Let $d=1$. Let $l \in \mathbf{N}$ and let $n_{1}<n_{2}<\cdots<n_{l}$ be an ascending sequence of integers and $x_{1}, \ldots, x_{l}$ another sequence of integers. It suffices to show that

$$
\operatorname{cap}\left(\left\{\left(n_{1}, x_{1}\right),\left(n_{2}, x_{2}\right), \ldots,\left(n_{l}, x_{l}\right)\right\}\right) \geq K \sqrt{l} .
$$

This, however, readily follows from $P_{n}(x) \leq a / \sqrt{n+1}$ by adopting $\mu$ that charges each point $\left(n_{j}, x_{j}\right)$ with unit mass. In fact $\sum_{j} G\left(\left(n_{k}, x_{k}\right),\left(n_{j}, x_{j}\right)\right)$ is less than $\sum_{j \geq k} a / \sqrt{n_{j}-n_{k}+1} \leq \sum_{j \geq k} a / \sqrt{j-k+1} \leq 3 a \sqrt{l}$, proving the required inequality.

For the multidimensional case, we also let $\mu$ be uniformly distributed with unit mass for each point. We have to show that $\sum_{\eta \in A} G(\xi, \eta) \leq$ const. $\sqrt{N}$ for $\xi \in A$. To 
this end we decompose $A$ into three parts according to $\xi=(n, x): A=A_{1} \cup A_{2} \cup A_{3}$, where

$$
\begin{aligned}
& A_{1}:=\{(m, y) \in A: m<n+\sqrt{N}\}, \\
& A_{2}:=\{(m, y) \in A: \sqrt{N}+n \leq m \leq N,|y-x| \leq \sqrt{m-n}\}, \\
& A_{3}:=\{(m, y) \in A: \sqrt{N}+n \leq m \leq N,|y-x|>\sqrt{m-n}\} .
\end{aligned}
$$

We let $n=0$ for simplicity, the arguments being identical for all $n \leq N$. Clearly $\sum_{\eta \in A_{1}} G(\xi, \eta) \leq \sqrt{N}$. By the local limit theorem in Section 3 we have

$$
\begin{aligned}
\sum_{\eta \in A_{2} \cup A_{3}} G(\xi, \eta) \leq & 3 \sum_{(m, y) \in A_{2} \cup A_{3}} p(m, y-x) \\
& +C_{1} \sum_{(m, y) \in A_{3}}\|y-x\|^{-2} m^{1-d / 2}(\log m)^{-p} .
\end{aligned}
$$

By a little reflection we realize that the first sum on the right side is dominated by a constant multiple of the sum of $p(m, y)$ over the set

$$
\left\{\left(m,\left(0, y^{(2)}, \ldots, y^{(d)}\right)\right): \sqrt{N} \leq m \leq N,\left|y^{(i)}\right| \leq C \sqrt{N}(i=2, \ldots, d)\right\}
$$

since the function $p(m, y)$ is decreasing with respect to $\|y\|$. Now by summing up $p(m, y)$ over $y$ first, the problem is reduced to the one-dimensional case and one can easily see that the first sum on the right side of (8.2) is dominated by const. $\sqrt{N}$. As for the second sum, we need to make different calculations according to the dimension. If $d=2$, the cardinality of the section $\left\{y:(m, y) \in A_{3}\right\}$ is at most const. $\sqrt{N}$. By estimating $\|x-y\|$ by $\sqrt{m}$ from below, the second sum is then bounded by const. $\sqrt{N}\left(\sum_{m=2}^{\infty} 1 / m(\log m)^{p}\right)$ as desired. If $d=3$ or 4 , we estimate the second sum from above, as in the case of the first sum, by

$$
\sum_{\sqrt{N} \leq m \leq N} \sum_{y \in \mathbf{Z}^{d-1}: 1 \leq|y| \leq d C \sqrt{N}}|y|^{-2} m^{1-d / 2}(\log m)^{-p} .
$$

The inner sum is roughly $\int_{1}^{\sqrt{N}} r^{d-4} d r$, and one finds that the double sum above is bounded by const. $\sqrt{N}$. In the case $d \geq 5$ we turn back to the sum $\sum_{\eta \in A_{3}} G$ and further decompose $A_{3}$ into two parts according as

$$
\|y-x\| \leq \sqrt{m \log m} \quad \text { or } \quad\|y-x\|>\sqrt{m \log m} .
$$

The contribution to $\sum_{\eta \in A_{3}} G$ from the first case, computed as in the case when $d=3$ or 4 , admits the required bound of const. $\sqrt{N}$. The contribution from the second case is dominated by

$$
\sum_{\sqrt{N} \leq m \leq N} P\left\{\left\|S_{m}\right\|>\sqrt{m \log m}\right\} \leq C_{2} \sum_{\sqrt{N \leq m \leq N}} \frac{1}{\sqrt{m}} \leq 2 C_{2} \sqrt{N} .
$$

Here we applied a remainder estimate for the central limit theorem that may read as follows: if the $Y_{n}$ 's are normalized sums of i.i.d. $\mathbf{R}$-valued random variables having a finite third moment, then $\left|P\left\{Y_{n} \geq u\right\}-(2 \pi)^{-1 / 2} \int_{u}^{\infty} \exp \left(-t^{2} / 2\right) d t\right|=O(1 / \sqrt{n})$ uniformly for $u \in \mathbf{R}$ ([GK]). The proof of Lemma 8 is complete.

Lemma 9. Let $d \geq 3$. Put $A(r, N):=\{(n, x): 0 \leq n \leq N,|x| \leq r\}$. Then for each $a>0, \operatorname{cap}(A(r, N)) \asymp r^{d-2} N$ uniformly for $N \geq a r^{2}$ as $r \rightarrow \infty$. 
Proof. To obtain the upper bound, we put for $\eta=(m, y)$

$$
\mu(\eta):= \begin{cases}1 & \text { if } \quad m=N \text { and }|y| \leq r \\ 1 / r & \text { if } \quad 0 \leq m<N \text { and } r-\sqrt{d} \leq|y| \leq r, \\ 0 & \text { otherwise. }\end{cases}
$$

Then $\sum \mu(\eta) \asymp r^{d-2} N$. We must therefore show that there exist $C$ and $r_{1}$ such that if $r \geq r_{1}, \sqrt{N} \geq c r$ and $\xi=(n, x) \in A(r, N)$, then $\sum_{\eta \in A(r, N)} G(\xi, \eta) \mu(\eta) \geq C$. But this is readily seen by applying the local limit theorem: in fact, if $n>N-r^{2}$, only the contribution of $\mu$ from the top end of $A(r, N)$ is enough to produce this lower bound, and if $n \leq N-r^{2}$, only that from the part of the side wall within the parabolic region $\left\{(m, y):|y-x|^{2} \leq 2(m-n)\right\}$ is enough.

For the lower bound of the capacity it suffices to show that the capacity of the hyper-plate $\left\{(n, x) \in A(r, N): x^{(1)}=0\right\}$ is bounded below by const. $r^{d-2} N$. The proof is easily done with a uniform distribution.

Complementary to Lemma 9, we have the following

Lemma 10. Put $A(r, \Delta, N):=\{(n, x): 1 \leq n \leq N, r \leq|x| \leq r+\Delta\}$. Then, for each $a>0, \operatorname{cap}(A(r, \Delta, N)) \asymp r^{d-1} \Delta$ uniformly for $r \geq 0$ and $\Delta>a \sqrt{N}$ as $N \rightarrow \infty$.

Proof. Let $\mu$ charge only the top end of $A(r, \Delta, N)$ with unit mass for each point of it, and apply the central limit theorem to see that $c \leq \sum_{\eta \in A(r, \Delta, N)} G(\xi, \eta) \mu(\eta)$ $\leq 1$, where $c$ is a positive constant that is independent of $\xi \in A(r, \Delta, N)$, and you obtain the result of the lemma since the cardinality of the top end of $A(r, \Delta, N)$ is bounded below as well as above by constant multiples of $r^{d-1} \Delta$.

\section{APPENDiX}

We give for completeness a proof of Theorem 8, in which we incidentally show that $S_{n} \in D_{p}$ except a finite number of $n$ (a.s.).

Proof of Theorem 8. We can suppose $h(n) \rightarrow \infty$ and $h^{2}(n) / n \rightarrow 0$, the latter owing to the strong law of large numbers. We define a sequence $\left(t_{m}\right)_{m=1}^{\infty}$ of positive integers inductively as follows. Choose $t_{1}$ large enough that $n / h^{2}(n)>1$ for $n \geq t_{1}$. With $t_{m}$ given, define $t_{m+1}$ by

$$
t_{m+1}-t_{m} \leq t_{m} / h^{2}\left(t_{m}\right)<t_{m+1}-t_{m}+1
$$

Take $p^{\prime}$ such that $1<p^{\prime}<p$. We apply the following relations:

$$
P\left\{\exists n \leq \nu,\left\|S_{n}\right\| \geq \sqrt{\nu} r\right\} \leq C P\left\{\left\|S_{\nu}\right\| \geq \sqrt{\nu} r\right\} \asymp r^{d-2} \exp \left(-r^{2} / 2\right),
$$

where the (first) inequality holds for all positive $r$ and $N$ and the (second) asymptotic relation is valid uniformly for $1<r \leq \sqrt{2 p^{\prime} \log \log \nu}$ as $\nu \rightarrow \infty$. The first one is obtained by a simple application of the strong Markov property of $S_{n}$ (and the central limit theorem) and the second one is an immediate consequence of Corollary 3 in Section 3. Now suppose (7.6a) holds. Put

$$
r_{m}:=\inf \left\{h(n): t_{m} \leq n \leq t_{m+1}\right\} \cdot \sqrt{t_{m} / t_{m+1}} .
$$


Then $r_{m} \geq\left(h^{2}\left(t_{m}\right)-C\right)^{1 / 2} /\left(1+1 / h^{2}\left(t_{m}\right)\right)^{1 / 2} \geq\left(h^{2}\left(t_{m}\right)-C^{\prime}\right)^{1 / 2}$ owing to $(9.1)$ and (7.6a). Hence

$$
\begin{aligned}
& P\left\{t_{m} \leq \exists n \leq t_{m+1},\left\|S_{n}\right\| \geq f(n), S_{n} \in D_{p}\right\} \\
& \leq P\left\{\exists n \leq t_{m+1},\left\|S_{n}\right\| \geq \sqrt{t_{m+1}} r_{m}, S_{n} \in D_{p}\right\} \\
& \leq C_{1} \exp \left(-\frac{h^{2}\left(t_{m}\right)}{2}\right) \frac{h^{d}\left(t_{m}\right)}{t_{m}}\left(t_{m+1}-t_{m}\right) \\
& \leq C_{2} \sum_{n=t_{m-1}}^{t_{m}} \exp \left(-\frac{h^{2}(n)}{2}\right) \frac{h^{d}(n)}{n}
\end{aligned}
$$

(both (9.1) and (7.6a) are employed again for each of the last two inequalities). By taking $f(n)=\sqrt{2 p^{\prime} n \log \log n}$, for which the sum of the last member above over $m$ is finite and we do not need to impose the condition $S_{n} \in D_{p}$ in the first two lines of (9.2), we see that $S_{n} \in D_{p}$ eventually as $n \rightarrow \infty$ with probability one. This in turn proves the claim of the theorem for general $f$. In the case when (7.6b) is assumed, we have only to interchange the roles of $t_{m}$ and $t_{m+1}$ and to replace the limits of the last sum with $t_{m+1}, t_{m+2}$. The proof of Theorem 8 is complete.

Remark 5. Except for our choice of $t_{m}$ defined by (9.1) the argument made above is standard (cf. [F3, Sections 5 and 7 of Chapter 8]). We have another proof (a little more involved than that given above) that is suggested by Theorem 4 . In that approach the determination of $t_{m}$ by (9.1) is very natural: the point is that $t_{m+1}-t_{m}$ is the same order as the height of the set $U_{j}^{k}$ that contains $\left(t_{m}, f\left(t_{m}\right)\right)$ (use (6.1)). This fact makes it easy to compute the capacities of $A_{m}$ 's determined by means of $t_{m}$ (see Lemma 10) and provides a proof of Theorem 8 based only on the relation (2.3) and an elementary geometry of $U_{j}^{k}$ that may be summarized by (6.1) and (6.2). Feller [F1] has already used a relation similar to (9.1) for the determination of $t_{m}$ in the proof of his generalized version of Kolmogorov's test (for both directions), but he did not discuss the case when $h$ is not monotone.

\section{REFERENCES}

[E] Erdös, P., On the law of the iterated logarithm, Ann. of Math. 43 (1942), 419-436. MR 4:16j

[EG] Evans, L.C., Gariepy, R.F., Wiener's criterion for the heat equation, Arch. Rat. Mech. Anal. 78 (1982), 293-314. MR 83g:35047

[F1] Feller, W., The general form of the so-called law of the iterated logarithm, Trans. Amer. Math. Soc. 54 (1943), 373-402. MR 5:125c

[F2] Feller, W., The law of the iterated logarithm for identically distributed random variables, Ann. Math. 47 (1946), 631-638. MR 8:214h

[F3] Feller, W., An introduction to probability theory and its applications, Vol. I, 2nd ed., John Wiley and Sons (1957). MR 19:466a

[GK] Gnedenko, B.V., Kolmogorov, A.N., Limit distributions for sums of independent random variables (translated from Russian), Addison-Wesley, Reading, MA, 1954. MR 16:52d

[GL] Garofalo, N., Lanconelli, E., Wiener's criterion for parabolic equations with variable coeffcients and its consequences, Trans. Amer. Math. Soc. 308 (1988), 811-836. MR 89k:35104

[IM] Ito, K., McKean, H.P., Potentials and the random walk, Illinois J. Math. 4 (1960), 119-132. MR 22: 12317

[Ld] Landis, E.M., Necessary and sufficient conditions for regularity of a boundary point in the Dirichlet problem for the heat-conduction equation, Soviet Math. 10 (1969), 380-384. MR 41:7308 (of Russian original)

[Lm] Lamperti, J., Wiener's test and Markov chains, J. Math. Anal. Appl. 6 (1963), 58-66. MR 26:817 
[Lv] Lévy, P., Théorie de l'addition des variables aléatoires, Paris: Gautier-Villars (1937).

[M] Motoo, M, Proof of the law of iterated logarithm through diffusion equation, Ann. Inst. Statis. Math. 10 (1959), 21-28. MR 20:4331

[P] Petrovskii, I., Zur ersten Randwertaufgabe der Wärmeleitungsgleichung, Compos. Math. 1 (1935), 383-419.

[S] Spitzer, F., Principles of random walk, 2nd ed., Springer-Verlag (1976). MR 52:9383

[U] Uchiyama, K., A probabilistic proof and applications of Wiener's test for the heat operator, Math. Ann. 283 (1989), 65-86. MR 90b:60099

Department of Applied Physics, Tokyo Institute of Technology, Meguro-ku, Tokyo 152 , JAPAN

E-mail address: uchiyama@neptune.ap.titech.ac.jp 